\title{
Enfermedad de Lyme crónica vs. Síndrome Post-tratamiento Lyme - a propósito de un caso en el ámbito laboral de Navarra
}

\author{
Chronic Lyme Disease vs. Lyme Post-treatment Syndrome - About a Case in the \\ Navarra Workplace
}

\author{
Ignacio Pascual Osinaga' ${ }^{1}$ Asan Iknurov Mollov² \\ 1. Servicio de Prevención de Riesgos Laborales, Departamento de Presidencia, Función Pública, Interior y Justicia, \\ Pamplona, Navarra, España. \\ 2. Unidad Docente de Medicina Del Trabajo de Navarra, Pamplona, España.
}

Recibido: 31-11-2019

Aceptado: 03-12-2019

\section{Correspondencia}

Asan Mollov

C/Irunlarrea $484^{\circ} \mathrm{D} 31008$ Pamplona, Navarra, España

Tifn:673702394

Correo electrónico: mollov.asan@gmail.com

Resumen

Resumen: La enfermedad de Lyme es causada por la espiroqueta Borrelia Burgdoferi, que se transmite por la mordedura de la garrapata del género Ixodes. El diagnostico se realiza por ELISA y se confirma mediante Western blot o PCR. Una infección por Borrelia no solo desencadena una respuesta inmune humoral, sino que también activa la respuesta inmune celular medida por los linfocitos T, de allí la existencia del análisis Elispot-LTT, cual según la literatura científica tiene mayor especificidad y sensibilidad que incluso el Western blot. El presente caso de cuadro clínico atípico en un bombero con Enferemdad de Lyme y co-infección por Rickettsia conorii y Bartonella Heselae. Se declaró como enfermedad profesional dado que se adquirió en el ámbito laboral.

Med Segur Trab (Internet). 2019;65(257):285-291

Palabras clave: Enfermedad de Lyme, eritema crónico migrans, medicina del trabjo, exposición ocupacional

Abstract

Abstract: Lyme disease is caused by the spirochete Borrelia Burgdoferi, which is transmitted by the bite of the tick of the genus Ixodes. The diagnosis is made by ELISA and confirmed by Western blot or PCR. A Borrelia infection not only triggers a humoral immune response, but also activates the cellular immune response measured by the T lymphocytes, hence the existence of the Elispot-LTT analysis, which according to the scientific literature has greater specificity and sensitivity than even the Western blot. The

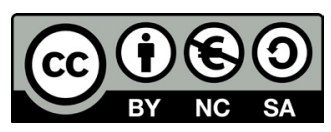

https://creativecommons.org/licenses/by-nc-sa/4.0/ 
present case of an atypical clinical picture in a firefighter with Lyme disease and co-infection with Rickettsia conorii and Bartonella Heselae. It was declared as a occupational disease since it was acquired in the workplace.

Med Segur Trab (Internet). 2019;65(257):285-291

Keywords: Lyme disease, Erithema chronicum migrans, Occupational Medicine, occupational exposure 
La borreliosis de Lyme (Enfermedad de Lyme-EL) es enfermedad multiorgánica con manifestaciones dermatológicas, reumáticas, neurológicas y cardíacas cuya lesión más específica, desde el punto de vista diagnóstico, es el eritema crónico migratorio. La EL está producida por espiroquetas del complejo Borrelia burgdorferi sensu lato. En Europa este complejo incluye 3 genoespecies patógenas para el hombre, B. burgdorferi sense stricto, B. afzelii y B. garinii. El diagnóstico se basa en el cuadro clínico y pruebas serológicas. Las pruebas pueden apoyar la sospecha, pero deben ser valoradas en contexto clínico por su alta incidencia de falsos negativos en pacientes con enfermedad temprana. Respecto a las pruebas serológicas, desde 1995 la recomendación de la CDC Atlanta está basada en una estrategia de dos pasos. Se realiza de primera instancia un ELISA, seguido de un Western inmunoblot (WB) en caso de ser positiva la primera prueba. La sensibilidad de la prueba de ELISA está determinada por el tiempo en el cual se realiza. Por ejemplo, en pacientes con enfermedad temprana pueden no detectarse anticuerpos contra $B$. burgdorferi, llevando a resultados falsos negativos. Cuando se realiza el WB dentro de las primeras cuatro semanas de inicio de la enfermedad, se recomienda realizar tanto IgM como IgG. Test diagnósticos en sangre ELISPOT y citometría de flujo de CD57 en ocasiones ayudan en el diagnóstico de casos atípicos ${ }^{1}$.

Se considera apropiado iniciar tratamiento con una probabilidad preestudios alta, basándose en síntomas y signos después de mordedura por garrapata del género Ixodes. Borrelia burgdorferi es sensible a penicilinas, cefalosporinas, tetraciclinas y macrólidos, no se han descrito resistencias secundarias. En función de la fase de la enfermedad y de las manifestaciones clínicas se utiliza una pauta $\mathrm{u}$ otra $^{2}$. El tratamiento de elección en el adulto es doxiciclina. El ensayo clínico de Stupica D. et al. de $2012^{3}$ ha dejado demostrado que 10 días de tratamiento con $100 \mathrm{mg} / 12 \mathrm{~h}$ de doxiciclina es suficientes para tratar el Eritema migrans en Europa. No existen vacunas comercializadas para la profilaxis de la EL, y el mejor método para prevenir esta afección es evitar la picadura y en caso de producirse, retirada temprana entre $24-48 \mathrm{~h}^{4}$.

Es el caso de un varón de 44 años, bombero con antecedentes personales de Síndrome de Waterhouse-Friederchsen en el contexto de meningitis en la infancia y resto sin interés. El cuadro presente se inició a finales de julio de 2016 cuando durante la jornada laboral en la zona del Pirineo Navarro, sufrió una picadura de garrapata en la zona periumbilical, que él mismo, al final de su jornada de $24 \mathrm{~h}$ extrajo sin aparente dificultad y sin dejar restos del artrópodo en la piel. Varios días después empezó el desarrollo de un cuadro clínico atípico con superposición de etapas y co-infección con diferentes bacterias variando en el tiempo presentado en la Figura 1 de forma cronología, prosiguiendo al mismo tiempo la clínica, pruebas de serología y el tratamiento durante 24 meses.

Durante el proceso diagnostico se le realizaron TAC craneal, TAC toraco-abdominopelvico, RNM encefálica, dos punciones lumbares, electromiograma, electroneurograma, electrocardiograma transtoracico, ECG, radiografía de tórax todos ellos sin hallazgos significativos. Ultramicroscopia en campo oscuro (15 meses tras la picadura), mostró pocos leucocitos y linfocitos, pero se observaron espiroquetas a las $48 \mathrm{~h}$ y sospecha de crecimiento de parásitos, es uno de los métodos de diagnóstico directo de espiroquetas (Leptosira spp., Borrelia spp., Treponema spp., Brachyspira spp., etc...). No es posible saber a qué familia filogenética pertenece la presencia de espiroquetas, pero indica la necesidad de estudios posteriores para tratar de identificarlas.

Alrededor del $2^{\circ}$ mes de la picadura se le extirpó lesión de la zona de la picadura y es analizó por el Anatomía patológica con resultado de granuloma por cuerpo extraño. Aproximadamente durante el $7^{\circ}$ mes desde la picadura apareció una lesión sugestiva de eritema migratorio que fue fotografiada en el Servicio de Prevención (Imagen 1), lesión eritematosa anular con zona pálida central de unos $10 \mathrm{~cm}$ y bultoma sobre la cicatriz tras la extirpación del granuloma por cuerpo extraño. El bultoma fue extirpado con resultado histológico de pseudolinfoma T. 
Figura 1. Cronología del cuadro clínico durante 24 meses (Clínica, Serología, Tratamiento).

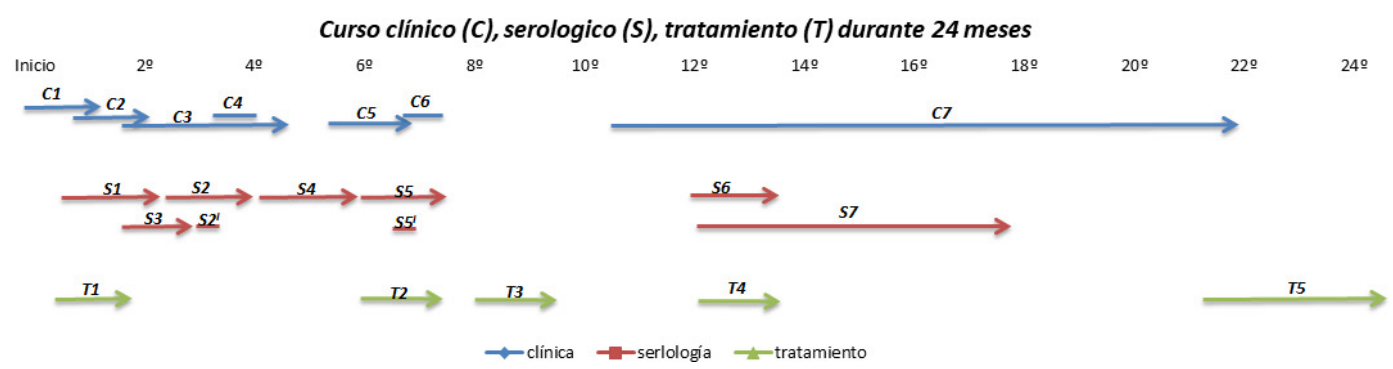

Clínica: C1 Cuadro pseudogripal (febrícula de 38 C, distermia, hiperhidrosis, odinofagia y congestión nasal). C2 C1 + síntomas de malestar general, astenia y debilidad en ambas piernas. $\mathbf{C 3} \mathrm{Cl}+\mathrm{C} 2+$ dolor en cara palmar de ambos antebrazos más intenso en el lado izquierdo, acompañado de parestesias y hipoestesias en hemicara izquierda, labio superior y que se extendieron al resto de la hemicara hasta la frente y cuero cabelludo, así como en los pulpejos de los dedos, diaforesis, astenia e hiporexia. C4 Dos episodios de mareo con giro de objetos, nauseas, cefaleas con sensación de embotamiento opresiva retroorbitaria bilateral, una caída sin pérdida de conocimiento, persistencia de febrícula, mialgias, artralgias y dolor abdominal. C5 Malestar general, cansancio, debilidad muscular grave, alteraciones digestivas (nauseas, vértigos y mareos, falta de apetito), pérdida de peso unos $5-6 \mathrm{~kg}$, pesadez en brazos y piernas, cefalea holocraneal tipo opresiva con reagudizaciónes que coinciden con picos de febrícula y a menudo termina en cefaleas intensas, dolores articulares sobre todo en tobillos. C6 Aparece una lesión sugestiva de eritema migratorio (Imagen 1). C7 Malestar general, astenia, distermia, hiperhidrosis nocturna, polimialgias, parestesias en extremidades izquierdas y hemifacial izquierda, cefaleas holocraneales opresiva, disminución de concentración y pérdida de memoria reciente.

Serología: S1 Borrelia burgdoferi lgG neg. S2 S1 + Fiebre Botonosa Mediterránea-IFI (positivo), VIH-neg. S2' LCR-Rickettsia spp. (PCR-hibridación) neg. (Centro Nacional de Microbiología). S3 Anaplasma spp., virus de la fiebre hemorrágica CrimeaCongo y PCR de Babesia spp. en sangre todos ellos con resultado neg. S4 Lyme-Borrelia burgdoferi lgG neg, Sifilis- neg, Brucella Rosa de Bengala-neg, quantiferon neg, VIH neg, FQ Elisa lgG y lgM neg, Fiebre Botonosa Mediterránea IFI 1/160- positiva, punción lumbar (citobioquímica-normal, PCR de Borrelia en LCR neg, cultivo sin crecimiento). S5 ELISPOT y CD 57 Citometría de flujo (Alemania) (Tabla 1). S5' LCR-Borrelia (PCR- hibridación) neg. (Centro Nacional de Microbiología). S6 ELISPOT (España) (Tabla 1). S7 Borrelia burgdoferi lgG neg., Brucella Rosa de bengala neg., Ebstein Barr neg., Toxoplasma lgG neg., VHB, VIH, Fiebre Q, QF-neg., Bartonella henselae y quintana $\lg G$ positivo, Rickettsia Conorii positivo.

Trafamienfo: T1 Doxiciclina $100 \mathrm{mg}$ cada 12h/4 semanas. T2 Ceftiaxona i.v. 5 días por semana durante 6 semanas, Azitromicina v.o., Ursobilane v.o, Artemisiam y Serrapentasa. T3 Penicilina i.m 3 días, Azitromicina, Doxiciclina, Tinidazol. T4 Gentamicina i.v y Rifampicina v.o. T5 Amoxicilina, Azitromicina y Tinidazol (3 meses)

Imagen 1. Lesión sugestiva de eritema migratorio presentada por el caso en el $7^{\circ}$ mes tras la picadura

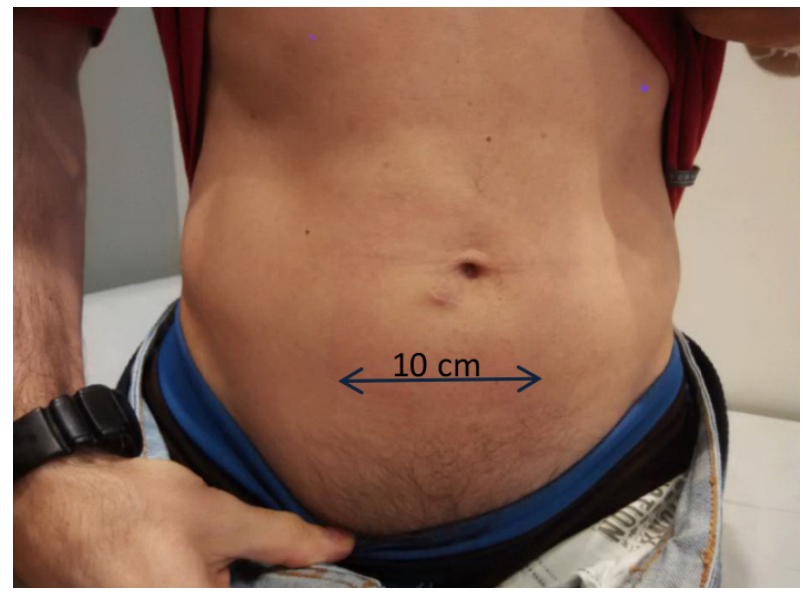

El diagnóstico serológico es más complicado en Europa debido a la presencia de múltiples genoespecies de $B$. burgdorferi, por lo que las pruebas serológicas deben incluir antígenos de las diferentes genoespecies o antígenos compartidos 5 .

Al principio los especialistas coinciden en la sospecha de una borreliosis no complicada. Dado el correcto tratamiento inicial tras la picadura y después de descartar neuroborreliosis el neurólogo cambió el diagnostico a posible Síndrome post tratamiento Lyme, por la persistencia de síntomas y la tórpida evolución del cuadro. El mejor indicador de neuroborreliosis activa es LCR con características inflamatorias, pleiocitosis linfocítica 
y respuesta intratecal de anticuerpos específicos contra $B$. burgdorferi ${ }^{6}$. Los pacientes con neuropatía periférica no presentan signos meníngeos ni producción intratecal de anticuerpos ${ }^{7}$.

La prueba de inmunoblot no fue realizada, por lo tanto un resultado negativo de ELISA no excluyó el diagnostico. Como el resultado del test ELISA fue negativo (en cuatro ocasiones), se le solicitaron tests diagnósticos en sangre ELISPOT y citometría de flujo de CD57 para descartar borreliosis en laboratorios independientes de Alemania en el $6^{\circ}$ mes y de España en el $12^{\circ}$ mes.

Los resultados del ELISPOT- test (Tabla 1) indicaron una actividad celular contra Borrelia burgdoferi. La citometría de flujo de CD57 indicó una situación de imunosupresión crónica, situación que puede ser causada por la infección por B. burgdoferi o por alguna otra bacteria como la Chlamydia/Mycopasma pneumoniae. Los números bajos de las células CD+ T se puede ver en las infecciones por EBV, CMV, HSV, HHVG, Coxsackie. Según el resultado se llegó a la conclusión que el paciente sufre Enfermedad de Lyme crónica. Una infección de borrelia no solo desencadena una respuesta inmune humoral (Ac), sino también activa la respuesta inmune celular (linfocitos T). El ELISPOT-LTT test es altamente sensible y puede detectar incluso una sola de las células T-Borrelia reactiva en sangre. Existen numerosas referencias bibliográficas en la literatura científica donde se demuestra que el ELISPOT para Borrelia Spp. tiene mayor especificidad y sensibilidad que incluso la técnica de WB para el diagnóstico de EL en fases evolucionadas. El numero bajo de CD57 indica hasta qué punto está suprimido el sistema inmune a causa de la enfermedad. En estadios avanzados prueba de ello es la disminución de células NK/CD3-, CD56+ y la disminución del número total de las células NK activadas CD3-, CD56+, $\operatorname{CD} 57+^{8,9,10}$.

Tabla 1. Resultado ELISPOT Y CD 57 Citometría de flujo $6^{\circ}$ y $12^{\circ}$ mes

\begin{tabular}{|c|c|c|c|c|}
\hline \multicolumn{4}{|c|}{$6^{\circ}$ mes (Alemania) } & \multirow{2}{*}{$\begin{array}{c}12^{\circ} \text { mes (España) } \\
\text { ELISPOT }\end{array}$} \\
\hline ELISPOT & CD 57 Citc & etría de flujo & & \\
\hline $\begin{array}{l}\text { Borrelia Burgdoferi } \\
\text { Fully Antigen: }+18 \\
\text { (normal <2) } \\
\text { - Borrelia Burgdoferi Osp- } \\
\text { Mix: }+6 \text { (normal <2) } \\
\text { - Borrelia Burgdoferi LFA- } \\
\text { 1: } 0 \text { (normal <2) }\end{array}$ & $\begin{array}{l}\text { Test CD3+ (\%): } \\
\text { Test CD3+ (absolute): } \\
\text { NK cells CD56 CD3 } \\
(\%) \\
\text { NK cells CD56 CD3 } \\
\text { (absolute) } \\
\text { CD 57+ NK- cells (\%) } \\
\text { CD57+ NK- cells } \\
\text { (aboslute) }\end{array}$ & $\begin{array}{c}69 \% \\
(-) 658 / \mathrm{ul} \\
18,76 \% \\
179 / \mathrm{ul} \\
20,42 \% \\
-37 / \mathrm{ul}\end{array}$ & $\begin{array}{c}62-80 \\
900-1900 \\
6-29 \\
62-80 \\
2-77 \\
100-300\end{array}$ & $\begin{array}{l}\text { - OPS-mix/basal: } 20 \\
\text { (positivo) } \\
\text { - Borrelia B31/basal: } 50 \\
\text { (positivo) } \\
\text { - Recuento de cell. NK } \\
\text { CD57+: } 17 \text { celulas/ } \\
\text { mcgL }\end{array}$ \\
\hline
\end{tabular}

La determinación del antígeno de Lyme en orina, la tinción inmunofluorescente para formas deficientes de la pared celular de B. burgdorferi, la PCR en sangre u orina para la detección de ADN de B. burgdorferi, las pruebas de transformación de linfocitos (LTT) y la determinación de la disminución de la respuesta de linfocitos CD57, no han sido aprobadas por ninguna agencia o sociedad científica como válidas para el diagnóstico y por este motivo, están desaconsejadas en estos $\operatorname{casos}^{11,12}$.

La comunicación de pacientes con síntomas inespecíficos (como fatiga crónica, dolores articulares y musculares etc ) después del tratamiento de la EL es conocido como Síndrome de la enfermedad de Lyme después del tratamiento o "Post-Treatment Lyme Disease Syndrome". El término "Enfermedad de Lyme crónica" se ha utilizado para describir a personas con diferentes síntomas, también se usa para describir síntomas en pacientes que no presentan evidencia de una infección actual o pasada por B. burgdorferi. Debido a la confusión sobre cómo se emplea el término "Enfermedad de Lyme crónica", los expertos no respaldan su uso ${ }^{13}$.

El trabajador fue valorado en varias ocasiones por Enfermedades infecciosas, quienes confirmaron serología positiva para Rickettsia conorii. Son bacterias de crecimiento 
estrictamente intracelular que se transmiten a través de vectores artrópodos a diferentes mamíferos que actúan como reservorios entre los que se encuentran los seres humanos. Producen cuadros clínicos de gravedad variable, pero suelen incluir la triada de fiebre cefalea y erupción cutánea (5-20 días tras la picadura). En 75\% de los casos se produce una lesión ulcerada de bordes ligeramente elevados con un fondo negruzco rodeado de un área eritematosa.

12 meses tras la picadura el trabajador sufrió una co-infección por Bartonella Heselae (enfermedad por arañazo de gato), que precisó ingreso hospitalario donde se confirmó selrologicamente (IgG Bartonella Heselae positivo) y se le proporció tratamiento con Gentamicina i.v y Rifampicina v.o.

Finalmente se concluyó que el trabajador padece al menos dos enfermedades relacionadas con la picadura de garrapata (borreliosis o enfermedad de Lyme y rickettsiosis o fiebre exantemática mediterránea), cuales tuvieron lugar durante el trascurso de su actividad laboral.

Patologías derivadas de la picadura de garrapata figuran en el cuadro de enfermedades profesionales con las correspondientes codificaciones del Real Decreto 1299/2006, de 10 de noviembre ${ }^{14}$. Tras un proceso complejo en el que estuvieron implicadas varias instituciones al trabajador se le reconoció la contingencia profesional del proceso vía judicial.

El bombero pasó temporalmente a servicios auxiliares estando limitado para tareas que supongan esfuerzo físico intenso o prolongado y manipulación manual de cargas características de bombero operativo.

Usar ropas protectoras en áreas de riesgo, repelentes y eliminar precozmente las garrapatas, que requieren un tiempo de adherencia mínimo de $48 \mathrm{~h}$ para transmitir la enfermedad siguen siendo los métodos más útiles para la profilaxis. La enfermedad de Lyme poco prevalente en nuestro medio y endémica en otros países, puede ver incrementada su incidencia como consecuencia del aumento de los viajes internacionales.

\section{REFERENCIAS BIBLIOGRÁFICAS}

1. Aránzazu Portillo, Sonia Santibáñez, José A. Oteo. Enfermedad de Lyme. Enferm Infecc Microbiol Clin. 2014;32(Supl 1):37-42

2. Wormser GP, Dattwyler RJ, Shapiro ED, Halperin JJ, Steere AC, Klempner MS, et al. The clinical assessment, treatment, and prevention of Lyme disease, human granulocytic anaplasmosis, and babesiosis: clinical practice guidelines by the Infectious Diseases Society of America. Clin Infect Dis2006;43:1089-134.

3. Stupica D, Lusa L, Ruzić-Sabljić E, Cerar T, Strle F. Treatment of erythema migrans with doxycycline for 10 days versus 15 days. Clin Infect Dis. 2012;55:343-50.

4. Oteo JA, Blanco JR, Ibarra V. ¿Podemos prevenir las enfermedades transmitidas por garrapatas? Enferm Infecc Microbiol Clin. 2001;19:509-13.

5. Marques A. Chronic Lyme Disease: A Review. Infect Dis Clin N Am. 2008;22:341-60

6. Pfister H-W, Rupprecht TA. Clinical aspects of neuroborreliosis and post-Lyme disease syndrome in adult patients. Int J Med Microbiol. 2006;296 Suppl 40:11-6.

7. Blaauw AAM, Rijpkema SGT, Kuiper H, Bijlsma JWJ. Lyme disease: who should be tested and treated, and how? Neth J Med. 1997;51:154-62

8. Jin C, Roen DR, Lehmann PV, Kellermann GH An Enhanced ELISPOT Assay for Sensitive Detection of Antigen-Specific T Cell Responses to Borrelia burgdorferi. Cells. 2013 Sep 13;2(3):607-20. doi: 10.3390/ cells2030607.

9. Nordberg M, Forsberg P, Nyman D, Skogman BH, Nyberg C, Ernerudh J, Eliasson I, Ekerfelt C Can ELISPOT Be Applied to A Clinical Setting as A Diagnostic Utility for Neuroborreliosis? Cells. 2012 Jun 8;1(2):153-67. doi: 10.3390/cells1020153.

10. Nielsen CM, White MJ, Goodier MR, Riley EM. Functional Significance of CD57 Expression on Human NK Cells and Relevance to Disease. Front Immunol. 2013 Dec 9;4:422. doi: 10.3389/fimmu.2013.00422. 
11. Klempner MS, Schmid CH, Hu L, Steere AC, Johnson G, McCloud B, et al. Intralaboratory reliability of serologic and urine testing for Lyme disease. Am J Med. 2001;110:217-9.

12. Marques A, Brown MR, Fleisher TA. Natural killer cell counts are not different between patients with post-Lyme disease syndrome and controls. Clin Vaccine Immunol. 2009;16:1249-50.

13. Feder HM Jr, Johnson BJ, O'Connell S, Shapiro ED, Steere AC, Wormser GP et al. A critical appraisal of "chronic Lyme disease". N Engl J Med. 2007 Oct 4;357(14):1422-30.

14. Real Decreto 1299/2006, de 10 de noviembre, por el que se aprueba el cuadro de enfermedades profesionales en el sistema de la Seguridad Social y se establecen criterios para su notificación y registro.

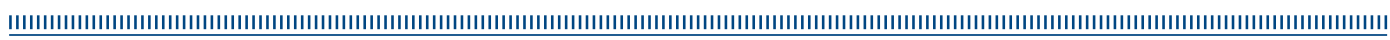

